\title{
ON THE ORIGIN OF S0 GALAXIES
}

\author{
Uta Fritze - v. Alvensleben \\ Universitätssternwarte Göttingen, Geismarlandstr. 11, 37083 Göttingen, Germany
}

\begin{abstract}
I will review the basic properties of S0 galaxies in the local Universe in relation to both elliptical and spiral galaxies, their neighbours on the Hubble sequence, and also in relation to dwarf spheroidal (dSph) galaxies. This will include colours, luminosities, spectral features, information about the age and metallicity composition of their stellar populations and globular clusters, about their ISM content, as well as kinematic signatures and their implications for central black hole masses and past interaction events, and the number ratios of S0s to other galaxy types in relation to environmental galaxy density.

I will point out some caveats as to their morphological discrimination against other classes of galaxies, discuss the role of dust and the wavelength dependence of bulge/disk light ratios. These effects are of importance for investigations into the redshift evolution of S0 galaxies - both as individual objects and as a population. The various formation and transformation scenarios for S0 and $\mathrm{dSph}$ galaxies will be presented and confronted with the available observations.
\end{abstract}

Keywords: S0 galaxies, formation and evolution

\section{Introduction}

Until some years ago, S0 galaxies were thought to be simple and wellunderstood: old passively evolving purely stellar systems in which everything had happened in the distant past, which are boring now and bound to go on fading into a dark and unspectacular future. In recent years a series of surprising observations led to a vividly renewed interest in S0 galaxies, their formation, transformation and evolution.

\section{Conventional Wisdom and Recent Puzzles}

Located on the Hubble sequence between Ellipticals (Es) and Spirals (Sps) because of their strong $\mathrm{r}^{1 / 4}$-bulges and their small and smooth exponential disks with Bulge-to-Total light ratios $\mathbf{B} / \mathbf{T} \sim 0.6$ in the B-band, S0 galaxies hold keys to 3 fundamental issues: 1. does Hubble's classification describe a continuous sequence from Es through $\mathrm{Sd}$ and Irr galaxies with S0s being the transition types or are Es and Sps fundamentally different and, if so, where 
do the S0s belong? 2. What determines the properties of S0 galaxies: initial conditions or environmental effects, nature or nurture? 3. The rate of evolution of S0 (and E) galaxies can test cosmological structure formation scenarios and constrain their parameters.

Classically, S0 galaxies were thought to have formed in a monolithic initial collapse with most of their Star Formation (SF) on a short timescale in the early Universe. Their relatively unimportant stellar disks (as compared to spirals) formed from leftover or accreted gas shortly after the bulge as both components do contain red stellar populations, little gas and essentially no SF. S0 galaxies follow relations established for ellipticals, like the colormagnitude relation, the black-hole mass - velocity dispersion, Faber-Jackson, Fundamental Plane (FP), and luminosity-metallicity relations. They also follow the Tully-Fisher relation for spirals, although with larger scatter. The observations that S0s are the dominant galaxy population in the central regions of nearby galaxy clusters while scarce in clusters at intermediate redshift and in the field provided a major trigger into renewed interest in the S0s and their formation or transformation histories. Recent detections of unexpected amounts of gas, dust and SF, kinematic peculiarities, fine-structure, inward central color gradients, etc. added to this new interest. Various scenarios have been suggested for the transformation accompanying the infall into the gradually forming galaxy clusters of the spiral rich field galaxy population into the S0-, dSph-, and dE-rich population of todays rich clusters. Timescales for the various aspects of the transformation have been discussed and possible progenitors or early stages of these transformation processes are being looked for in high and intermediate redshift clusters. Probably more than one evolutionary path has led to the observed manifold of S0 and dSph systems.

\section{S0s on the Hubble Sequence}

S0 follow many trends along the Hubble sequence originally defined by morphological appearance, i.e. the bulge component getting less prominent and the disk component and spiral structure getting more pronounced from early to late-type galaxies: the colors of S0s are almost as red on average as those of ellipticals and redder than those of spirals that get increasingly bluer towards later Hubble types, their spectra, as well, have properties that fit in between those of Es and Sa's. The content in neutral and molecular gas in most S0s is low to negligible similar to ellipticals while it increases systematically towards later spiral types. IRAS though has detected $50 \%$ of all E and S0 galaxies (Jura et al. 1987), later investigations revealed that cold gas is particularly common among peculiar S0s that also show relatively high $\mathrm{HI}$ content and $\mathrm{H}_{\alpha}$. HI structures and kinematics often suggest an external origin. Dust masses from FIR measurements are typically a factor $\sim 10$ higher 
than those from optical extinction, indicating that the dust distribution is more extended than that of the star light. CO and $\mathrm{HI}$ are more strongly concentrated in the centers than for spirals (Bregman et al. 1998, Pogge \& Eskridge 1993, Sadler et al. 2000, Welch \& Sage 2003). However, even the most gas-rich S0s contain much less gas than expected if they had formed all their stars in an early burst and evolved as a closed-box since then (Faber \& Gallagher 1976). As opposed to massive ellipticals, S0s do not show luminous X-ray halos. Presentday star formation rates $\mathrm{SFR}_{o}$ are low in most $\mathrm{S} 0 \mathrm{~s}$, their ratio of time averaged past SFR $\langle\mathrm{SFR}\rangle$ to present-day $\mathrm{SFR}_{o}$ falls between the respective values for Es and the earliest type spirals (e.g. Sandage 1986). Their SF efficiences, i.e. SFRs normalised to gas content, are higher than for spirals, for which they decrease further from Sa through Sd. In terms of average luminosity and $\mathrm{M} / \mathrm{L}-$ ratio, S0s also range between Es and Sa's.

All these observations indicate that the Hubble sequence is a continuous sequence, not only in terms of $\mathrm{B} / \mathrm{T}$-light ratios, but also in terms of colors and spectral properties, gas content, $\mathrm{SFR}_{o},\langle\mathrm{SFR}\rangle$, and, hence, stellar population ages. On the other hand, one might look at the S0s in the light of the apparent dichotomy among ellipticals: Low-luminosity Es are fast rotators, have powerlaw surface brightness profiles towards the resolution limit, disky isophotes, and approximately isotropic velocity dispersions, all compatible with the idea that dissipation has played a role in their formation and dynamical evolution. Luminous Es, however, show slow or no rotation, are triaxial, have cores, boxy isophotes, and anisotropic velocity dispersions (Kormendy \& Illingworth 1982). Kormendy \& Bender (1996) redefine the Hubble sequence as extending from boxy Es via disky Es to S0s, Sa, ..., Sd, Irr. van den Bergh (1994) argued in favor of the S0s sharing the dichotomy among the ellipticals with faint S0s being flattened and prolate and bright S0s being the ones that are truely intermediate between Es and Sa's. Faber et al. 's (1997) observations that, with one exception (NGC 524), S0s all have power-law surface brightness profiles support this view.

It has long been known that in terms of central parameters (central surface brightness, central velocity dispersion) and absolute luminosity SOs and bulges follow the relations among ellipticals while dwarf Ellipticals (dEs) and Globular Clusters follow different relations in the famous Kormendy (1985) diagrams. From these diagrams and the knowledge from stellardynamical modelling that mergers cannot increase the central phase space density by more than a factor of two, it is clear that S0s or normal/giant Es cannot be produced in mergers of dEs or dSphs. Ryden et al. (1999) found no distinct structural differences between $\mathrm{dEs}$ and $\mathrm{dSphs/dS0s.}$

S0s harbour nuclear black holes like bulges and Es, as revealed by absorption or, in some cases, emission line analyses of high spatial resolution HST spectra. The two S0s in Pinkney et al. 's (2003) sample follow the black hole 
mass - velocity dispersion relation $\mathrm{M}_{\mathrm{BH}}-\sigma_{\mathrm{e}}$ for Es and bulges found by Gebhardt et al. (2000) and Tremaine et al. (2002). If all S0s have central BHs still seems to be an open question, e.g. in view of NGC 524's core.

S0s follow the central $\mathrm{Mg}_{2}-\sigma_{o}$ relation of ellipticals and have metallicity gradients shallower by factors $2-3$ than spirals, therewith fitting into the trend of increasing metallicity gradients from early to late-type spirals. In contrast to spirals and ellipticals, however, many S0s apparently get older from inside out, i.e. their bulge stellar populations are younger than those of their disks. S0s follow the anticorrelation between $\mathrm{EW}\left(\mathrm{H}_{\beta}\right)$ and velocity dispersion $\sigma_{o}$ of ellipticals - indicating that more massive galaxies have older stellar populations (Fisher et al. 1996). The age of a stellar population in this context means the time elapsed since the last epoch of significant SF. Concerning the relative stellar population ages in the disks and bulges of field S0s, observations have revealed discrepant results: BJHK photometry with surface brightness profile decomposition of 35 S0s showed that because of similar $J-K$ and $H-K$ colors both components probably have similar metallicities and the different $B-H$ colors indicate that disks are $\sim 3-5$ Gyr younger than bulges. Some S0s show evidence for AGB light contributions implying active SF until $2-3$ Gyr ago (Caldwell 1983, Bothun \& Gregg 1990). NGC 7332 which has emission line gas kinematically decoupled from the stellar component and an Atype bulge spectrum (Bertola et al. 1992, Fisher et al. 1994, Hibbard \& Rich 1990), indicative of recent accretion and starburst events, is found by Bender \& Paquet (1999) to have a bulge star population spectroscopically older than its disk population. NGC 5102 and NGC 404, resolved into individual stars with HST, both show blue inward color gradients and significant populations of young stars $\gtrsim 15$ and $\gtrsim 300 \mathrm{Myr}$, respectively, in their centers (Deharveng et al. 1997, Tikhonov et al. 2003). Investigations into stellar population ages of $33 \mathrm{~S} 0 \mathrm{~s}$ and $19 \mathrm{Es}$ in the Coma cluster over a wide range in luminosities $-20.5 \leq \mathrm{M}_{\mathrm{B}} \leq-17.5$ showed that $1 . \geq 40 \%$ of the S0s (and none of the Es!) had significant SF in their central regions during the last $5 \mathrm{Gyr}$ and that 2. the fraction of S0s with recent SF increases with decreasing luminosity (Poggianti et al. 2001a). This luminosity dependence of the luminosity weighted ages is independently found from optical and NIR photometry of S0s in Abell 2218 $(\mathrm{z}=0.17)$ by Smail et al. (2001). It explains the discrepant results of earlier studies and support the idea of a dichotomy between low and high-luminosity SOs.

S0s as well as ellipticals in Virgo, Coma and other nearby clusters - both rich and poor - follow a Color-Magnitude Relation (CMR) in the sense that luminous galaxies are redder than fainter ones (Bower et al. 1992, Andreon 2003). This CMR is primarily due to higher metallicities in brighter galaxies, as evidenced by spectroscopic observations yielding similar relations between $\mathrm{Mg}_{2}$ and luminosity or $\mathrm{Mg}_{2}$ and $\sigma$. In addition to metallicity differ- 
ences, age differences may also contribute. E.g. Kuntschner \& Davis (1998) and Kuntschner (2000) find from line strength analyses of Fornax Es and S0s that the luminous Es \& S0s have formed their stars earlier or stopped their SF earlier than the lower luminosity ones. The tightness of the CMR is conventionally assumed to imply uniform old stellar population ages and, within hierarchical galaxy formation scenarios, that more massive galaxies formed earlier from more massive building blocks.

Schweizer et al. (1990) found fine structure, i.e. ripples, shells, plumes, boxiness, X-structure, etc. in $\sim 50 \%$ of the field S0s. The amount of fine structure as quantified by his fine structure parameter $\Sigma$ correlates with deviations from the CMR towards bluer colors, with increasing $\mathrm{H}_{\beta}-\mathrm{EWs}$, and with decreasing $\mathrm{Mg}_{2}$ (Schweizer \& Seitzer 1992) in the sense that $\sim 50 \%$ of the field S0s had major mergers with significant starbursts about $3-8$ Gyr ago (Schweizer 1993, 1999).

E/S0s form a homologous 3-parameter family with low scatter described by the apparantly universal Fundamental Plane (FP) relation between effective radius $\mathrm{r}_{\mathrm{e}}$, velocity dispersion $\sigma$, and effective surface brightness $\left\langle\mathrm{I}_{\mathrm{e}}\right\rangle$ within $\mathrm{r}_{\mathrm{e}}$ (Djorgovski \& Davis 1987, Dressler et al. 1987, Jorgensen et al. 1993, 1996, Scodeggio et al. 1998a, b)

$$
\log \mathrm{r}_{\mathrm{e}}=\mathrm{a} \cdot \log \sigma+\mathrm{b} \cdot \log \left\langle\mathrm{I}_{\mathrm{e}}\right\rangle+\mathrm{c}
$$

The scatter is generally $\lesssim$ the measurement errors, smaller in clusters than in the field and strongest for the low-luminosity S0s (van Dokkum et al. 2001). In combination with the Virial Theorem the FP relations imply a weak mass dependence of the mass-to-light ratio $\mathrm{M} / \mathrm{L} \sim \mathrm{M}^{0.2}$ (Faber et al. 1987). A salient feature of the FP is that it indicates an intimite relation between spectral and dynamical evolution aspects of galaxies by coupling tightly structural and stellar population parameters. Observations of the FP in the NIR show that 1.) the scatter is independent of passband, implying that variations in age are balanced by variations in metallicity, and 2.) that the slope increases steadily from $\mathrm{U}$ through $\mathrm{K}$, pointing to systematic changes in metallicity and age and DM content/homology breaking (Pahre et al. 1998). Conventionally, the tight FP is interpreted in terms of E/S0s being very homogeneous old stellar systems. However, it has been shown that stellardynamical mergers conserve the FP and its small scatter (Capelato et al. 1995, Evstigneeva et al. 2004) and that E+Sp merger remnants soon after merging come to lie on a FP of changes that is only tilted by $15^{\circ}$ relative to the observed FP (Levine 1995). Observations by Lake \& Dressler (1986) of a dozend well-known Sp - Sp merger remnants from Arp's (1966) and Arp \& Madore's (1985) catalogues still featuring long tidal tails like NGC 7252 or NGC 3921 have shown that - very surprisingly - these objects $\leq 1 \mathrm{Gyr}$ after their merger and strong starburst well fit into the $\mathrm{L}-\sigma$ and $\mathrm{FP}-$ relations, casting serious doubt upon the idea that the very existence 
and the tightness of these relations necessarily imply uniform old ages for $\mathrm{E}$ and S0 galaxies. Apparently violent relaxation very fast transforms the inner regions within $1 \mathrm{r}_{\mathrm{e}}$.

S0 galaxies not only follow relations for elliptical galaxies, they also obey the Tully-Fisher Relation (TFR) for spirals: $\mathrm{L} \sim \mathrm{V}_{\max }^{\alpha}$ with $\alpha \sim 4$ and $\mathrm{V}_{\max }$ the maximum rotation velocity (Tully \& Fisher 1977, Pierce \& Tully 1992). Mathieu et al. (2002) find 6 S0s to follow the H-band TFR with small scatter $\sim 0.3$ mag while being offset by $\sim 1.1$ mag to fainter magnitudes in I, consistent with some fading after SF truncation (see below). Results are still somewhat controversial with Hinz et al. $(2001,2003)$ reporting a small offset $\leq 0.2$ mag from the H-band TFR and a large scatter for massive $\left(V_{c} \geq 200 \mathrm{kms}^{-1}\right)$ S0s in Virgo and Coma and Neistein et al. (1999) finding a smaller offset in I and a large scatter for 18 nearby field S0s, in particular at the faint end. The luminosity range of the sample, like for the stellar population ages, or the field versus cluster environment might be a key to reconcile the apparently discrepant results. Note that rotation curves are much more difficult to measure for S0s than for spirals because they largely have to rely on absorption lines. Moreover, about $50 \%$ of the Virgo S0s show weird rotation curves that do not allow to derive a $\mathrm{V}_{\max }$ at all (Rubin et al. 1999). Different formation paths may well lead to S0s with diverse properties.

\section{Classification Problems}

S0s are distinguished against Sa galaxies either by their less prominent and featureless disks or, in quantitative analyses, by some limiting B/T-light ratio. Face-on S0s are hard to distinguish against ellipticals, the only reliable method being long-slit absorption line spectroscopy that allows to detect an S0's rapidly rotating disk. In a magnitude-limited Coma sample Jorgensen \& Franx (1994) found only $12 \%$ Es among the huge E/S0 population. For distant clusters often only the combined E/SO population is considered. Using evolutionary synthesis models for bulge and disk components separately with two different SF histories - const. SFR as appropriate for disks and exponentially declining SFRs $\left(\mathrm{t}_{*} \sim 1\right.$ Gyr) for bulges - and combining bulges and disks to obtain after a Hubble time the observed average B-band B/T-light ratios of various Hubble types we could study both the wavelength and the redshift dependences of $\mathrm{B} / \mathrm{T}$-light ratios (Schulz et al. 2003). We investigate three different scenarios for the onset of bulge and disk SF: 1.) both start in the early universe at $\mathrm{z} \sim 3-5,2$.) bulge $\mathrm{SF}$ starts at $\mathrm{z} \sim 3-5$ and disk $\mathrm{SF}$ later at $\mathrm{z} \sim 1$, and 3.) disk $\mathrm{SF}$ starts at $\mathrm{z} \sim 3-5$ and bulge $\mathrm{SF}$ later at $\mathrm{z} \sim 1$.

We found a significant wavelength dependence of $\mathrm{B} / \mathrm{T}$-light ratios (Fig.1a): for $\mathrm{S} 0 \mathrm{~s} \mathrm{~B} / \mathrm{T}$ increases by a factor $2-3$ from $U$ through $K$ (for Sd's by a factor $3-4$ ) in agreement with $\mathrm{H}$-band $\mathrm{B} / \mathrm{T}$ - determinations by Eskridge $e t$ 

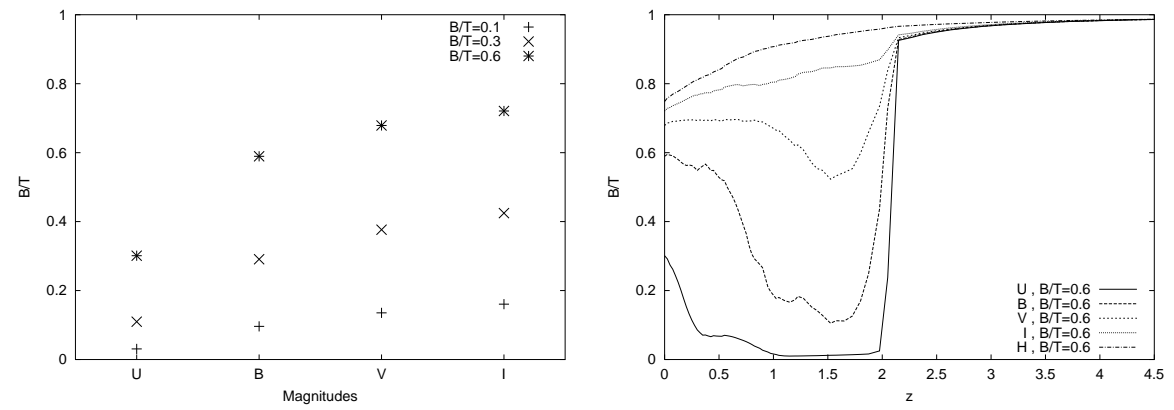

Figure 1. (a) Wavelength dependence, (b) redshift dependence of the B/T-light ratio for S0s. Bulge and disk SF both starting at $\mathrm{z} \sim 3-5$.

al. (2002). The amount of increase slightly depends on the relative ages of bulge and disk stars (cf. Schulz et al. for details). In addition, we found a strong redshift evolution of $\mathrm{B} / \mathrm{T}$-light ratios that cannot be compensated for by switching from $B$ to $V, R, I$ in a comparison with galaxies at redshifts $\mathrm{z}=0.3,0.5,0.7$. Models show that in any of the three scenarios $\mathrm{B} / \mathrm{T}-$ light ratios of S0s at higher redshift determined from surface profile decomposition in bands that correspond to restframe B are significantly overestimated. S0s at higher redshift therefore have a high chance to be misclassified as Es (Fig.1b). The reason is the difference in time evolution of the disk and bulge components due to their different SF histories. We therefore decided to give cosmological and evolutionary corrections separately for bulge and disk components and the 3 scenarios that we explored: bulge and disk of equal age, bulge older or younger than the disk (cf. Schulz et al. 2003). It is true that changes get smaller towards longer wavelengths and that therefore galaxy classification in I, as e.g. done for the HDF galaxies by Marleau \& Simard (1998), or in a NIR band is less affected. For S0s and Es, unfortunately, the NIR B/T-light ratios are very similar and do not allow to separate them from each other.

\section{Formation and Transformation of S0s}

In the central regions of local rich virialised galaxy clusters $\mathrm{S} 0$ galaxies are the dominant galaxy type, making up as much as $\sim 60 \%$ of the bright galaxy population. Dwarf galaxies (dEs, dSOs, dSphs) are about twice as numerous as the luminous ones in Coma (de Lucia et al. 2004). Distant clusters, on the other hand, contain increasing fractions of blue galaxies, as first discovered by Butcher \& Oemler $(1978,1984)$. Van Dokkum (2001) and Dahlen et al. (2004) find a factor $\sim 5$ increase in the blue galaxy fraction from $\mathrm{z} \sim 0.5$ to $\mathrm{z}=0$, i.e. over the last $5 \mathrm{Gyr}$ (using $\mathrm{H}_{\mathrm{o}}=65, \Omega_{\mathrm{m}}=0.3, \Omega_{\lambda}=0.7$ ). Most of 
the blue galaxies in distant clusters are Sps and Irrs with ongoing SF (Smail et al. 1997), some are having starbursts, others show Balmer absorption lines indicative of recent starburst (Dressler \& Gunn 1983). Some of the red galaxies also have strong Balmer absorption lines $(\mathrm{E}+\mathrm{A}, \mathrm{k}+\mathrm{a})$, i.e. are slightly older post-starbursts (spectroscopic BO-effect). Fasano et al. $(2000,2001)$ and Couch et al. (1998) show that while the fraction of ellipticals in the cluster population remains approximately constant from redshift $\mathrm{z} \sim 0.5$ to $\mathrm{z}=0$, the fraction of spirals decreases by a factor $\sim 5$, and the fraction of S0s increases by the same factor. This suggests a significant transformation of spirals into S0s from $\mathrm{z} \sim 0.5$ to $\mathrm{z}=0$, i.e. over the last 5 Gyr. At the same time, the faintto-luminous galaxy ratio in clusters increased from $\sim 1$ at $\mathrm{z}=0.75$ to $\sim 2$ at $\mathrm{z}=0$ (de Propris et al. 2003).

The field galaxy population also being spiral-rich and poor in S0s and Es, the transformations of spirals into S0s and of luminous into dwarf galaxies seem to be linked with processes during the continuous infall of field spirals towards increasingly rich clusters. Formation and evolution of galaxy clusters and Large Scale Structure hence appear to go hand in hand with morphological and spectral transformation of important parts of their galaxy population.

A large variety of formation and transformation scenarios have been proposed for S0s over the years and it seems that more than one of them is needed to account for the observed diversity of the S0 population. Which one(s) is (are) realised or prevalent may depend on environment and epoch; massive and low-mass S0s may have different formation and evolution histories.

Like bulges in later-type spirals the spheroidal componets of S0s could have formed in the classical fast initial collapse with rapid $S F$ scenario - at least for those $50 \%$ of the field S0s that do not show any fine structure or signs of central rejuvenation.

Internal instabilities in spiral disks can form bars that efficiently funnel gas and stars into the central parts and bars, in turn, can dissolve to form a bulge component. The huge bulges of S0s require several circles of bar formation and destruction as seen in numerical simulations (Combes et al. 1990, Raha et al. 1991). Depending on the relative amounts of gas and disk stars funneled into the central regions, stellar populations in the bulge might be older or younger than those in the disk.

Major spiral - spiral mergers have been shown to result in elliptical galaxies since the Toomres' pioneering work. Incomplete violent relaxations transforms stellar disks into de Vaucouleurs profiles while saving small gradients. The delayed and protracted backfall of HI from the tidal tails onto the main body, as e.g. observed in NGC 7252, can rebuild a gaseous disk on timescales of few Gyr that by and by transforms into a stellar disk, resulting in a disky elliptical or an S0 galaxy as seen in combined N-body and hydrodynamical simulations by Hibbard \& Mihos (1995) and in Barnes' (2002) models. Few 
Gyr after the global or nuclear starbursts triggered respectively in prograde and retrograde encounters (Bekki 1995) the merger remnants have been shown to reach typical S0 - and somewhat later elliptical - galaxy colors and spectra in our evolutionary synthesis models (Fritze - v. Alvensleben \& Gerhard 1994a, b). It is clear that this major merger scenario is only viable for massive and luminous S0s. Only in field or group environments galaxy encounter velocities are low enough for efficient merging. Hence the luminous S0s in clusters must have been preprocessed in the field or within infalling groups - in agreement with observations that luminous S0s have stellar population ages as old as ellipticals. In this scenario, the stellar population in the bulge will be older than that in the disk. Observations of the rich cluster MS 1054-03 at $\mathrm{z}=0.83$ reveal a substantial number of major mergers, $17 \%$ in total, not only in the center but also in the outer parts, consistent with the idea of enhanced merging in infalling groups (van Dokkum et al. 1999). Gavazzi et al. (2003) observationally caught a collective starburst among the members of a galaxy group falling into the cluster Abell 1387.

Minor mergers (e.g. mergers with galaxy mass ratios 3:1) or accretion events have been shown by Barnes (1996) and Bekki (1998) to be viable routes towards intermediate or low-luminosity S0s, again in the field or in groups rather than in dense clusters where galaxies have too high relative velocities. The nature and gas content of the objects involved determines the outcome. A $\mathrm{Sp}+\mathrm{dE}$ or a $\mathrm{Sp}+(\mathrm{d})$ Irr merger will result in an $\mathrm{S} 0$ with a bulge older or younger than the disk depending on the strength of a possibly triggered starburst.

Major mergers among gas-rich galaxies form populous systems of new star clusters, many of which are compact and strongly enough bound to survive for Gyrs (Schweizer 2002, Fritze - v. Alvensleben 1998, 1999). Their enhanced metallicities and younger ages, when determined from spectroscopy or multi-wavelength photometry, are much more precise tracers of past violent SF events than the integrated galaxy light (Anders et al. 2004a, Fritze v. Alvensleben 2004a, b). If the young star clusters forming in minor mergers are also long-lived still is an open question (Anders et al. 2004b, Fritze - v. Alvensleben 2004b). S0 galaxies with bimodal Globular Cluster (GC) color distributions in any case testify back to a major merger origin. Both the luminous S0 NGC 1380 (Kissler - Patig et al. 1997) and the low-luminosity S0 NGC 3115 show bimodal GC color distributions. The field stars in NGC 3115 also show the same bimodal color distribution (Elson 1997). Kundu \& Whitmore (2001) estimate that $\gtrsim 10-20 \%$ of the S0s have bimodal GC color distributions and, hence, a clear major merger origin. Age differences between the blue metal-poor and the red metal-rich GCs are small ( $\lesssim 3 \mathrm{Gyr}$ ). Hence, the mergers producing the red GC population in the starbursts they triggered must have happened early. On average, S0s have less populous GC systems 
than ellipticals of comparable luminosity and are closer to spirals in this respect, although with large scatter.

Harassment is an important process in dense cluster environments, where fast galaxy - galaxy encounters destabilise the disks of infalling spirals, drag out strong short-lived tidal tails and tear away stars from their outer disks, leaving the inner parts as $\mathrm{dE}$, low-luminosity $\mathrm{S} 0$ or $\mathrm{dSph}$ galaxies on timescales of few Gyr while releasing up to 50\% of the original stellar mass of the incoming spiral to the cluster potential (Moore et al. 1995, 1998). As the remnants of this process consist of the former central parts of much more massive galaxies, they are to be expected to deviate from the $\mathrm{Mg}_{2}-\sigma-$ relation towards too high metallicities for their mass. Poggianti et al. (2001b) report a significant population of low-luminosity galaxies in Coma with exactly these properties: enhanced metallicities for their luminosities. Apart from being harassment products they could also be Tidal Dwarf Galaxies, i.e. recycling galaxies forming out of gas and stars torn out into a tidal tail from a disk galaxy in interaction and becoming self-gravitating and dynamically independent there (cf. Duc \& Mirabel 1999, Weilbacher et al. 2002, 2003).

Beyond these different types of galaxy - galaxy interactions the presence of a hot dense ICM as observed at X-wavelengths in the centers of nearby rich clusters will affect the gaseous components of infalling spirals. Processes like ram pressure stripping or sweeping of their HI disks have been predicted as the density and pressure of the hot ICM are observed to be comparable or higher than those of the ISM within galaxies, and they can directly be seen in terms of increasingly truncated and displaced HI disks in spirals towards the center of the Coma cluster (Cayatte et al. 1990, Bravo - Alfaro et al. 2000). It is clear that SF in these anemic spirals (van den Bergh 1976) will also be truncated. If the instability intrigued by the rapid removal of considerable amounts of HI will lead to a starburst consuming all the molecular gas and part of the central $\mathrm{HI}$ in one shot before final SF truncation and if it can also affect the stellar configuration is less clear. Observations that the galaxy population in clusters starts to deviate from the field galaxy population in terms of their SF activity at unexpectedly large distances from cluster centers (around 3-4 $\mathrm{R}_{\mathrm{vir}}$ ) (Lewis et al. 2002, Gomez et al. 2003, Balogh et al. 2004, Gerken et al. 2004) raised the idea that the ICM densities that far out might still be high enough to drive away the low density gas from infalling galaxy halos that otherwise could serve as a reservoir for disk accretion and, hence, for SF (Larson et al. 1980, Bekki et al. 2002). If realised by nature, this halo gas stripping or starvation would lead to SF strangulation on a rather long timescale of order Gyr.

We have investigated the effect of SF truncation without or after a preceeding starburst on the photometric and spectral evolution of various spiral types in Bicker et al. (2002). Using evolutionary synthesis models that successfully describe undisturbed galaxies of types $\mathrm{Sa}, \mathrm{Sb}, \mathrm{Sc}$, and $\mathrm{Sd}$, we added bursts 
of various strengths and/or SF truncation at various evolutionary ages. Figs. $2 \mathrm{a}, \mathrm{b}$ show the time evolution of $(B-V)$ colors and of B-band luminosities $\mathrm{M}_{\mathrm{B}}$ for starbursts and/or SF truncation occuring at $\mathrm{z}=1$ in a $6 \mathrm{Gyr}$ old $\mathrm{Sc}$ galaxy. Unless the SFR does not go to zero, all models reach $\mathrm{S} 0$ galaxy colors fairly rapidly: within $2 \mathrm{Gyr}$ after SF truncation and within $4-5$ Gyr after a starburst, depending on its strength, in case of $B-V$. The luminosity evolution, of course, strongly depends on whether or not a starburst occured before SF truncation: between pure SF truncation and strong burst models there is a difference in final luminosity of 2 mag in $\mathrm{M}_{\mathrm{B}}$.
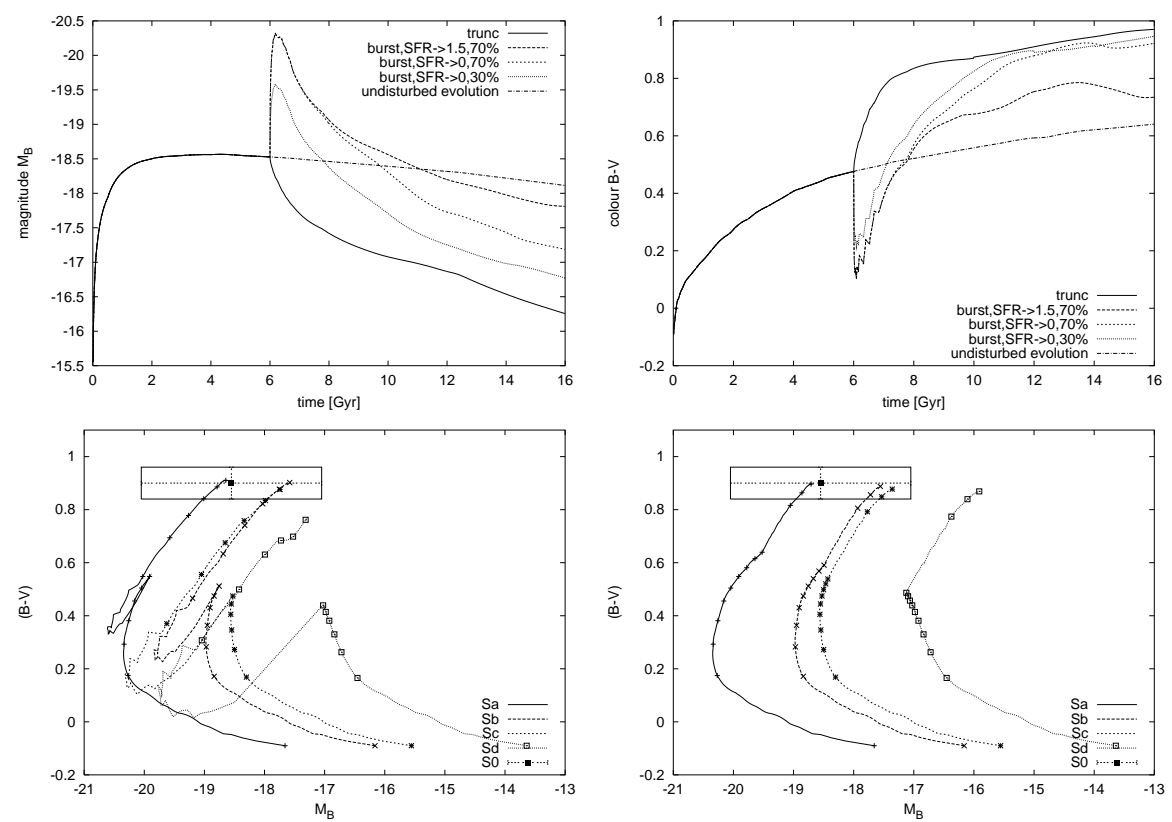

Figure 2. Luminosity (a) and color (b) evolution of an Sc spiral model with starbursts and/or SF truncation occuring at an age of $6 \mathrm{Gyr}$. CMD for various spiral models with starbursts occuring at $6 \mathrm{Gyr}$ (c) and with SF truncation at 9 Gyr (d). Ticks mark 1 Gyr steps. The box gives average observed $\mathrm{S} 0$ color and luminosity with $1 \sigma$ ranges.

The photometric evolution in $U B V R I J H K$ of these models is compared with observed S0 properties in terms of Color - Magnitude Diagrams (CMDs). Note that our undisturbed models are calibrated in mass as to yield after a Hubble time the average observed $\mathrm{M}_{\mathrm{B}}$ for the respective spiral type. As an example, Figs. 2c, d show in a CMD the evolution of various spiral types with strong bursts and/or SF truncation occuring at ages of $6 \mathrm{Gyr}$ and $9 \mathrm{Gyr}$, corresponding to redshifts $\mathrm{z}=1$ and $\mathrm{z}=0.5$, respectively. The box in the upper left indicates the observed average color and luminosity of S0s with their $1 \sigma$ ranges. About 4 Gyr after their strong starbursts $\mathrm{Sa}, \mathrm{Sb}, \mathrm{Sc}$ models all reach the color range 
of S0s, Sd models remain too blue. Only the Sa model reaches the average luminosity of S0s. Sb and Sc models after strong starbursts are able to account for the fainter S0s. Only 1.5 Gyr after SF truncation without a preceeding starburst all models show S0 galaxy colors, but again only the Sa progenitors reach average S0 luminosities, the Sb snd Sc progenitors turn into low-luminosity S0s, and Sd galaxies with truncated SF end up with luminosities of dSphs. SF truncation in $3 \mathrm{Gyr}$ young $\mathrm{Sa}$ and $\mathrm{Sb}$ progenitors, i.e. at $\mathrm{z}=2$, would produce galaxies too red for S0s. At such young ages, however, the dense hot ICM of today's rich cluster centers that is thought to be responsible for SF truncation by sweeping out the gas from infalling spirals might not yet have accumulated. We also considered mergers of equal spiral types that double the mass in stars and gas and the luminosity while not changing the colors. After strong bursts accompanying these mergers, Sa progenitors reach the bright end of the S0 galaxy luminosity distribution, and even Sd-galaxies, when merging with each other, reach average S0 luminosities. As far as the color evolution is concerned, our results are confirmed by an independent study by Shioya et al. (2002). Including also the luminosity evolution, however, our models allow for tighter constraints on the manifold of possible S0 progenitors than theirs. To investigate the recently renewed suggestion that SF strangulation by halo gas starvation might truncate SF on a longer timescale of $\geq 1$ Gyr (Larson et al. 1980, Bekki et al. 2002), we recalculated models under these conditions and we also analyse the spectral evolution, e.g. the evolution of emission and absorption lines (Fritze \& Bicker in prep.).

We find that SF truncation on a short timescale of order $10^{8} \mathrm{yr}$ is followed by a phase of moderate $\mathrm{H}_{\delta}$ - strength, while $\mathrm{SF}$ strangulation on a timescale of $10^{9}$ yrs does not develop enhanced $\mathrm{H}_{\delta}$ absorption. SF truncation after a preceeding burst results in an $\mathrm{H}_{\delta}-$ strong phase of $\sim 1.5 \mathrm{Gyr}$ duration (see also Barger et al. 1996, Poggianti et al. 1999, and Shioya et al. 2004). Note that red $\mathrm{H}_{\delta}-$ strong galaxies are also called $\mathrm{E}+\mathrm{A}$ or $\mathrm{k}+\mathrm{a}$ types.

We conclude that the progenitors of low-luminosity SOs can be Sa through $\mathrm{Sc}$ galaxies that experienced a starburst $>3 \mathrm{Gyr}$ ago, as well as $\mathrm{Sa} / \mathrm{Sb}$ galaxies with SF truncation at ages between 6 and 9 Gyr, i.e. at $1 \leq \mathrm{z} \leq 0.5$, or Sc galaxies with $\mathrm{SF}$ truncation as late as $\mathrm{z}=0.5$, i.e. after $9 \mathrm{Gyr}$ of undisturbed evolution. The strength of the burst does not make much difference. The progenitors of luminous S0s can only be early-type spiral - spiral or multiple mergers with starbursts occuring at ages $\leq 9$ Gyr, i.e. at $\mathrm{z} \geq 0.5$.

The progenitors of low- and high-luminosity S0s that had experienced a starburst have gone through an $\mathrm{H}_{\delta}-$ strong phase, first blue and then red, of $\sim 1.5$ Gyr duration. The progenitors of those low-luminosity S0s and dSphs that had their SF truncated without a starburst can, at maximum, have gone through a phase of intermediate-strength Balmer absorption provided the SF truncation happened on a short timescale of order $10^{8} \mathrm{yr}$. 
Barger et al. (1996) and Kodama \& Smail (2001) quantitatively investigated the possibility of a spiral-to-S0 transformation in terms of galaxy numbers and morphologies. Couch \& Sharples (1987) report a high proportion $(\sim 30 \%)$ of actively SFing and starbursting galaxies in 3 rich clusters at $\mathrm{z}=0.31$, many of which show signs of interactions while still having disk morphologies, and a high fraction of $\mathrm{H}_{\delta}-$ strong galaxies, most of which are regular spheroids, indicating that the timescale for the photometric transformation is somewhat shorter than that for the morphological transformation, but that the strong Balmer absorption features after strong starbursts still are detectable after the morphological transformation is accomplished. Maybe in mergers which are probably the origin of strong starbursts the morphological transformation is faster than for other transformation processes?

The decrease of the blue galaxy fraction towards $z=0$ probably is a consequence of the interplay between a decline in the galaxy infall rate with cosmic time, the overall decrease in gas content and SFR in the field galaxy population, and the increasing cluster richness together with the successive buildup of their dense hot ICM content. While at intermediate and high redshift, the starburst and E+A galaxies in clusters were bright high-mass objects, the actively SFing, starburst and postburst galaxies in local clusters are predominantly low-luminosity late-type dwarf galaxies. This trend is called downsizing effect (Bower et al. 1999, Poggianti 2004) and also reported for the field galaxy population (Cowie et al. 1996). Duc et al. (2002) conclude from ISOCAM mid-IR observations that up to $90 \%$ of the SF in a cluster $\mathrm{z}=0.18$ is hidden. If this would selectively apply for the most massive and metal-rich galaxies it might explain an apparent downsizing effect simply by a strong prevalence of dust at low redshift.

\section{Redshift Evolution of S0 Galaxy Populations}

Van Dokkum et al. (1998) investigate the redshift evolution of the CMR for $194 \mathrm{E} / \mathrm{S} 0$ galaxies in DL $1358+62$ at $\mathrm{z}=0.3$. They find the scatter, i.e. the age spread, for Es very small and independent of clustercentric radius $R_{\mathrm{cl}}$. The scatter for the S0s is equally small in the center, however, it increases considerably towards larger $\mathrm{R}_{\mathrm{cl}}$ with the $\mathrm{S} 0$ s being increasingly offset towards bluer colors at large $R_{\mathrm{cl}}$. This shows that while ellipticals have terminated their SF well before accretion, S0s stop SF in the outer parts of the cluster. Stanford et al. (1998) extend this study to 19 clusters at $\mathrm{z} \sim 0.9$ using NIR photometry and find the local slope conserved, the scatter, i.e. the apparent age spread among E/S0s still small and the degree of evolution independent of cluster richness or $\mathrm{L}_{\mathrm{X}}$. The fact that the slope for these young galaxy populations is not different from the local one implies that it results from a correlation of galaxy mass with metallicity, rather than with age. 
Studies of the redshift evolution of the FP aim at 3 aspects: the redshift evolution of its slope tells about size and mass evolution, i.e. about accretion and evolution in orbital structure, changes in the zero-point indicate evolution in $\mathrm{M} / \mathrm{L}$, due in part to the fading of the stellar population and possibly also affected by rejuvenation through accretion and SF, the redshift evolution of the scatter is a measure of the homogeneity of the E/S0 population at various

lookback times. Semianalytical $\Lambda \mathrm{CDM}$ structure formation models predict that high density environments should collapse first and fastest. Hence, they lead us to expect cluster galaxies to be older than field galaxies and therefore to expect differences in the redshift evolution of the FPs of field and cluster galaxies (Kauffmann 1996, Diaferio et al. 2001). Remember again that age in this context means time since the last epoch of significant SF.

The redshift evolution of the FP for cluster E/S0s in the redshift interval $0.3 \leq \mathrm{z} \leq 0.8$ as studied by Kelson et al. (1997), Bender et al. (1998), van Dokkum et al. (1998), Jorgensen et al. (1999), and Wuyts et al. (2004) indicates that the scatter remains close to local until $\mathrm{z} \sim 0.5$ and only increases at $\mathrm{z}=0.8$, the zero-point shift indicates $\sim 1$ mag brightening to $\mathrm{z}=0.8$. The evolution in $\mathrm{M} / \mathrm{L}$ is slow, implying a galaxy formation redshift $\mathrm{z}_{\mathrm{f}}>2.8$ consistent with results from the redshift evolution of the CMR. The redshift evolution of the FP for field galaxies was studied by means of Keck-LRes spectroscopy by Gebhardt et al. (2003) on 21 Es and 15 S0s from the DEEP sample in the redshift range $0.3 \leq \mathrm{z} \leq 1$ with $\langle\mathrm{z}\rangle=0.8$ and by van Dokkum \& Ellis (2003) on $10 \mathrm{E} / \mathrm{S} 0$ s from the HDF-N with $0.56 \leq \mathrm{z} \leq 1.02$. Both groups find slope and scatter similar to local, and a zero-point offset by 2.4 mag in $I$ (corresponding to rest-frame $B$ at $\mathrm{z}=1$ ), marginally in agreement with passive evolution of simple old populations. Comparison with the evolution of cluster E/S0s indicates an age difference between cluster and field E/S0s of less than $2 \mathrm{Gyr}$, which poses a problem for hierarchical structure formation models that predicted a larger age difference.

The redshift evolution of the TF relation remains inconclusive at present for the S0s due to the difficulties in measuring stellar velocity dispersions from absorption lines in high redshift spectra.

\section{Conclusions and Open Questions}

Located between ellipticals and spirals on the Hubble sequence, S0 galaxies play a key role in 3 fundamental and still largely open questions: as to the nature of Hubble's sequence, to the respective roles of nature versus nurture in shaping galaxy properties, and as valuable test cases for cosmological galaxy formation scenarios. Are S0s transition types on one continuous line from E through Sd galaxy types or do they belong to either of two fundamentally different classes of objects? I reviewed arguments for and against boths options 
and conclude that current evidence seems to favor a dichotomy among S0s similar to the one for ellipticals. In this sense, the luminous S0s have formed their stars earlier than the low-luminosity S0s and may have had different formation scenarios.

S0 galaxies share many properties with other dynamically hot stellar systems, as e.g. the relation between central black hole mass and velocity dispersion, the color-magnitude and Fundamental Plane relations, while also following the Tully-Fisher relation for spirals. We have seen that constraints from the FP and CMR on the ages and formation histories of S0s are less tight than originally thought, and that $50 \%$ of the field S0s show evidence for important intermediate age stellar contributions in their central regions together with morphological and kinematic traces of interactions events. Detections of HI, CO, HII, dust and SF activity in quite a number of S0s adds to this view. Together with the strong redshift evolution of the S0 and spiral fractions in galaxy clusters this provides evidence that nurture rather than nature, environmental effects rather than initial conditions have shaped S0 galaxies, that they are transformation rather than formation products. It is not clear at present if any classical early collapse + short timescale SF S0s do at all exist in the field - for sure not in clusters. It also seems clear that more than one transformation scenario must be at work to produce the observed manifold of S0s. Major spiral-spiral/Irr mergers with starbursts may result in luminous S0s, as shown by dynamical simulations as well as evolutionary spectral synthesis, with the protracted backfall of HI from the tidal tail(s) leading to the build-up of a secondary stellar disk on timescales of 3 Gyr. Minor mergers (3:1) or accretion events on the one hand, harassment, tidal stripping of stars and rampressure sweeping of gas on the other hand may result in lower-luminosity S0s and dSphs. Any kind of merging or accretion requires low relative velocities and, hence, is more probable within groups before or during their infall into clusters or in very early stages of cluster evolution. It may leave kinematic peculiarities, fine structure, and positive color gradients that can survive for a few Gyrs. If the bursts are strong enough the resulting new star cluster population with its age and metallicity may be better suited to trace back the SF history of its parent galaxy than the integrated light. Pixel-by-pixel analyses provide spatially resolved information about the respective contributions of old and younger stars. Harassment through fast galaxy-galaxy encounters is probably the dominant process in dense clusters, its end products will be low-luminosity S0s, dSphs or dEs that can be expected to deviate from the luminosity-metallicity relation because they are the leftover central parts of originally much more massive galaxies. Interactions with the dense hot X-ray emitting ICM in nearby rich galaxy clusters is observed to efficiently remove HI disks from spirals plunging into it, cutting their gas supply and, hence, truncating their SF while destabilising the stellar disks. The timescale for the 
morphological transformation seems to be longer than that for the photometric transformation, the strong Balmer absorption features after strong bursts, however, seem to survive the morphological transformation, at least in some cases.

If the much less dense halo gas is removed from spirals by the same process at larger cluster-centric radii already - with the effect of cutting the SFing disk off from its accretion reservoir causing SF strangulation on a relatively long timescale seems less clear. Detailed statistics of the various progenitor and transition types (normal and strong emission line galaxies, blue and red $\mathrm{H}_{\delta}-$ strong galaxies) in clusters of varying richness and degree of relaxation and at various cluster-centric radii should ultimately allow to identify the relative importance of all these different formation paths. The fact that the morphologydensity relation seems to be continuous from the densest cluster centers out to the field probably indicates that, beyond the direct interaction with the clusters' central ICM and potential, more localised processes, e.g. in groups, must also play an important role.

It seems for sure that the $\mathrm{S} 0$ galaxies and the questions as to their origin will keep us excited for years to come and may still hold further surprises.

\section{Acknowledgments}

I gratefully acknowledge generous travel support from the organisers of this conference without which I could not have attended.

\section{References}

Anders, P., Bissantz, N., Fritze - v. Alvensleben, U., de Grijs, R., 2004a, MN 347, 196

Anders, P., de Grijs, R., Fritze - v. Alvensleben, U., Bissantz, N., 2004b, MN 347, 17

Andreon, S., 2003, A\&A 409, 37

Arp, H., 1966, Atlas of Peculiar Galaxies

Arp, H., Madore, B. F., 1985, A Catalogue of Southern Peculiar Galaxies

Balogh, M., Eke, V., Miller, C. et al. , 2004, MN 348, 1355

Barger, A. J., Aragon - Salamanca, A., Ellis, R. S. et al. , 1996, MN 279, 1

Barnes, J. E., 1996, IAU Symp. 171, 191

Barnes, J. E., 2002, MN 333, 481

Bekki, K., 1995, MN 276, 9

Bekki, K., 1998, ApJ 499, 635

Bekki, K., Couch, W. J., Shioya, Y., 2002, ApJ 577, 651

Bender, R., Saglia, R. P., Ziegler, B. et al. , 1998, ApJ 493, 529

Bender, R., Paquet, A., 1999, Ap\&SS 267, 283

Bertola, F., Buson, L. M., Zeilinger, W. W., 1992, ApJ 401, L79

Bicker, J., Fritze - v. Alvensleben, U., Fricke, K. J., 2002, A\&A 387, 412

Bower, R. G., Lucey, J. R., Ellis, R. S., 1992, MN 254, 601

Bower, R. G., Kodama, T., Terlevich, A., 1998, MN 299, 1193

Bothun, G. D., Gregg, M. D., 1990, ApJ 350, 73

Bravo - Alfaro, H., Cayatte, V., van Gorkom, J. H., Balkowski, C., 2000, AJ 119, 580 
Bregman, J. N., Snider, B. A., Grego, L., Cox, C. V., 1998, ApJ 499, 670

Butcher, H., Oemler, A., 1978, ApJ 219, 18

Butcher, H., Oemler, A., 1984, ApJ 285, 426

Caldwell, N., 1983, ApJ 268, 90

Capelato, H. V., de Carvalho, R. R., Carlberg, R. G., 1995, ApJ 451, 525

Cayatte, V., van Gorkom, J. H., Balkowski, C., Kotanyi, C., 1990, AJ 100, 604

Combes, F., Debbasch, F., Friedli, D., Pfenniger, D., 1990, A\&A 233, 82

Couch, W. J., Sharples, R. M., 1987, MN 229, 423

Couch, W. J., Barger, A. J., Smail, I. et al. , 1998, ApJ 497, 188

Cowie, L. L., Songaila, A., Hu, E. M., Cohen, J. G., 1996, AJ 112, 839

Dahlen, T., Fransson, C., Ostlin, G., Naslund, M., 2004, MN 350, 253

Deharveng, J.-M., Jedrzejewski, R., Crane, P. et al. , 1997, A\&A 326, 528

de Lucia, G., Poggianti, B. M., Aragon - Salamanca, A. et al. , 2004, astro-ph/0404084

de Propris, R., Colless, M., Driver, S. et al. , 2003, MN 342, 725

Diaferio, A., Kauffmann, G., Balogh, M. L. et al. , 2001, MN 323, 999

Djorgovski, S., Davis, M., 1987, ApJ 313, 59

Dressler, A., Gunn, J. E., 1983, ApJ 270, 7

Dressler, A., Lynden - Bell, D., Burstein, D. et al. , 1987, ApJ 313, 42

Duc, P.-A., Mirabel, I. F., 1999, IAU Symp. 186, 61

Duc, P.-A., Poggianti, B. M., Fadda, D. et al. , 2002, A\&A 382, 60

Elson, R., 1997, MN 286, 771

Eskridge, P. B., Frogel, J. A., Pogge, R. W. et al. 2002, ApJS 143, 73

Evstigneeva, E. A., de Carvalho, R. R., Ribeiro, A. L., Capelato, H. V., 2004, MN 349, 1052

Faber, S. M., Dressler, A., Davies, R. L. et al. , 1987, Nearly Normal Galaxies, Springer, New York, p. 175

Faber, S. M., Tremaine, S. et al. , 1997, AJ 114, 1771

Fasano, G., Poggianti, B. M., Couch, W. J. et al. , 2000, ApJ 542, 673

Fasano, G., Poggianti, B. M., Couch, W. J. et al. , 2001, Ap\&SS 277, 417

Fisher, D., Franx, M., Illingworth, G., 1996, ApJ 459, 110

Fisher, D., Illingworth, G., Franx, M., 1994, AJ 107, 160

Fritze - v. Alvensleben, U., 1998, A\&A 336, 83

Fritze - v. Alvensleben, U., 1999, A\&A 342, L25

Fritze - v. Alvensleben, U., 2004a, A\&A 414, 515

Fritze - v. Alvensleben, U., 2004b, in The Young Local Universe, eds. A. Chalabaev, Y. Fukui, T. Montmerle, in press

Fritze - v. Alvensleben, U., Gerhard, O. E., 1994a, A\&A 285, 751

Fritze - v. Alvensleben, U., Gerhard, O. E., 1994b, A\&A 285, 775

Gavazzi, G., Cortese, L., Boselli, A. et al. , 2003, ApJ 597, 210

Gebhardt, K., Bender, R., Bower, G. et al. , 2000, ApJ 539, L13

Gebhardt, K., Faber, S. M., Koo, D. C. et al. , 2003, ApJ 597, 239

Gerken, B., Ziegler, B., Balogh, M. et al. , 2004, astro-ph/0403652

Gomez, P. L., Nichol, R. C., Miller, C. J. et al. , 2003, ApJ 584, 210

Hibbard, J. E., Mihos, C. J., 1995, AJ 110, 140

Hibbard, J. E., Rich, R. M., 1990, ESO/CTIO Workshop on Bulges of Galaxies, ESO Garching, p. 295

Hinz, J. L., Rix., H.-W., Bernstein, G. M., 2001, AJ 121, 683

Hinz, J. L., Rieke, G. H., Caldwell, N., 2003, AJ 126, 2622

Jorgensen, I., Franx, M., 1994, ApJ 433, 553

Jorgensen, I., Franx, M., Kjaergaard, P., 1993, ApJ 411, 34

Jorgensen, I., Franx, M., Kjaergaard, P., 1996, MN 280, 167 
Jorgensen, I., Franx, M., Hjorth, J., van Dokkum, P. G., 1999, MN 308, 833

Jura, M., Kim, D. W., Knapp, G. R. et al. 1987, ApJ 312, L11

Kauffmann, G., 1996, MN 281, 487

Kelson, D. D., van Dokkum, P. G., Franx, M. et al. , 1997, ApJ 478, L13

Kissler - Patig, M., Richtler, T., Storm, J., della Valle, M., 1997, A\&A 327, 503

Kodama, T., Smail, I., 2001, MN 326, 637

Kormendy, J., 1985, ApJ 295, 73

Kormendy, J., Bender, R., 1996, ApJ 464, L119

Kormendy, J., Illingworth, G., 1982, ApJ 256, 460

Kundu, A., Whitmore, B. C., 2001, AJ 122, 1251

Kuntschner, H., 2000, MN 315, 184

Kuntschner, H., Davis, R. L., 1998, MN 295, L29

Lake, G., Dressler, A., 1986, ApJ 310, 605

Larson, R. B., Tinsley, B. M., Caldwell, C. N., 1980, ApJ 237, 692

Levine, S., 1995, Interacting Galaxies, ed. G. Longo, p. 129

Lewis, I., Balogh, M., de Propris, R. et al. , 2002, MN 334, 673

Marleau, F. R., Simard, L., 1998, ApJ 507, 585

Mathieu, A., Merrifield, M. R., Kuijken, K., 2002, MN 330, 251

Moore, B., Katz, N., Lake, G. et al. , 1995, Nat 379, 613

Moore, B., Lake, G., Katz, N., 1998, ApJ 495, 139

Neistein, E., Maoz, D., Rix, H.-W., Tonry, J. L., 1999, AJ 117, 2666

Pahre, M. A., de Carvalho, R. R., Djorgovski, S. G., 1998, AJ 116, 1606

Pierce, M. J., Tully, R. B., 1992, ApJ 387, 47

Pinkney, J., Gebhardt, K., Bender, R. et al. , 2003, ApJ 596, 903

Poggianti, B. M., Smail, I., Dressler, A. et al. , 1999, ApJ 518, 576

Poggianti, B. M., Bridges, T. J., Carter, D. et al. , 2001a, ApJ 563, 118

Poggianti, B. M., Bridges, T. J., Mobasher, T. J. et al. , 2001b, ApJ 562, 689

Poggianti, B. M., Bridges, T. J., Yagi, M. et al. , 2004, IAU Symp. 195, in press

Pogge, R. W., Eskridge, P. B., 1993, AJ 106, 1405

Raha, N., Sellwood, J. A., James, R. A., Kahn, F. D., 1991, Nat 352, 411

Rubin, V. C., Waterman, A. H., Kenney, J. D. P., 1999, AJ 118, 236

Ryden, B. S., Terndrup, D. M., Pogge, R. W. et al. , 1999, ApJ 517, 650

Sadler, E. M., Oosterloo, T. A., Morganti, R., Karakas, A., 2000, AJ 119, 1180

Sandage, A., 1986, A\&A 161, 89

Shioya, Y., Bekki, K., Couch, W. J., de Propris, R., 2002, ApJ 565, 223

Shioya, Y., Bekki, K., Couch, W. J., 2004, ApJ 601, 654

Schulz, J., Fritze - v. Alvensleben, U., Fricke, K. J., 2003, A\&A 398, 89

Schweizer, F., 1993, Dynamics and Interactions of Galaxies, Springer, p. 60

Schweizer, F., 1999, Ap\&SS 267, 299

Schweizer, F., 2002, IAU Symp. 207, 630

Schweizer, F., Seitzer, P., Faber, S. M. et al. 1990, ApJ 364, L33

Schweizer, F., Seitzer, P., 1992, AJ 104, 1039

Scodeggio, M., Giovanelli, R., Haynes, M. P., 1998a, AJ 116, 2728

Scodeggio, M., Giovanelli, R., Haynes, M. P., 1998b, AJ 116, 2738

Smail, I., Dressler, A., Couch, W. J. et al. , 1997, ApJS 110, 213

Smail, I., Kuntschner, H., Kodama, T. et al. , 2001, MN 323, 839

Tikhonov, N. A., Galazutdinova, O. A., Aparicio, A., 2003, A\&A 401, 863

Terlevich, A. I., Caldwell, N., Bower, R. G., 2001, MN 326, 1547

Tremaine, S., Gebhardt, K., Bender, R. et al. , 2002, ApJ 574, 740

Tully, R. B., Fisher, J. R., 1977, A\&A 54, 661 
van den Bergh, S., 1976, ApJ 206, 883

van den Bergh, S., 1994, AJ 107, 153

van den Bergh, S., 2004, ApJ 601, L37

van Dokkum, P. G., Ellis, R. S., 2003, ApJ 592, L53

van Dokkum, P. G., Franx, M., Kelson, D. D. et al. , 1998, ApJ 500, 714

van Dokkum, P. G., Franx, M., Fabricant, D. et al. , 1999, ApJ 520, L95

van Dokkum, P. G., Franx, M., Kelson, D. D., Illingworth, G. D., 2001, ApJ 553, L39

Weilbacher, P. M., Fritze - v. Alvensleben, U., Duc, P.-A., Fricke, K. J., 2002, ApJ 579, L79

Weilbacher, P. M., Duc, P.-A., Fritze - v. Alvensleben, U., 2003, A\&A 397, 545

Welch, G. A., Sage, L. J., 2003, ApJ 584, 260

Wuyts, S., van Dokkum, P. G., Kelson, D. D. et al. , 2004, ApJ 605, 677 